SOUNDING BOARD

\title{
HOW CAN WE DEVELOP A MORE CLINICALLY USEFUL AND ROBUST ALGORITHM FOR DIAGNOSING AND TREATING NOCTURIA? ICI-RS $2017^{1}$
}

Everaert $\mathrm{K}^{1, *}$, Hervé F1,*, Bower W2, Djurhuus J3, Dmochowski R4, N. Fines, Wein A6, Wyndaele M7, Weiss J5

${ }^{1}$ Department of Urology, Ghent University Hospital, Ghent, Belgium.

*Equal study contribution

2Department of Medicine and Community Care, Melbourne Health; Faculty of Medicine, Dentistry and Health Science, University of Melbourne, Australia.

3Department of Clinical Medicine, Aarhus University, Aarhus, Denmark.

${ }^{4}$ Department of Urology, Vanderbilt University, Nashville, Tennesse; USA.

5Department of Urology, SUNY Downstate College of Medicine, New York, USA.

${ }^{6}$ Department of Urology, Perelman School of Medicine - University of Pennsylvania, Philadelphia, USA.

${ }^{7}$ Bristol Urological Institute, Southmead Hospital, Bristol, United Kingdom.

Corresponding author. François Hervé (ORCID 0000-0002-9079-251X)

Urology department, Ghent University Hospital

De Pintelaan 185, 9000 Ghent, Belgium.

E-mail adress: francois.herve@uzgent.be

Telephone number: 0032 (0) 93321351

Fax number: 0032 (0) 93323889

WORDCOUNT: 5893

\section{HOW CAN WE DEVELOP A MORE CLINICALLY USEFUL AND ROBUST ALGORITHM FOR DIAGNOSING AND TREATING NOCTURIA? ICI-RS 2017}

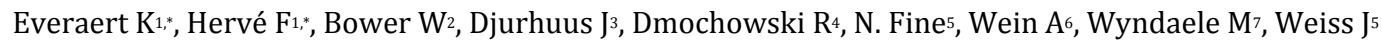

\begin{abstract}
1 This is the author manuscript accepted for publication and has undergone full peer review but has not been through the copyediting, typesetting, pagination and proofreading process, which may lead to differences between this version and the Version of Record. Please cite this article as doi:10.1002/nau.23569
\end{abstract}

This article is protected by copyright. All rights reserved. 
${ }^{1}$ Department of Urology, Ghent University Hospital, Ghent, Belgium.

*Equal study contribution

${ }^{2}$ Department of Medicine and Community Care, Melbourne Health; Faculty of Medicine, Dentistry and Health Science, University of Melbourne, Australia.

${ }^{3}$ Department of Clinical Medicine, Aarhus University, Aarhus, Denmark.

${ }^{4}$ Department of Urology, Vanderbilt University, Nashville, Tennesse; USA.

${ }^{5}$ Department of Urology, SUNY Downstate College of Medicine, New York, USA.

${ }^{6}$ Department of Urology, Perelman School of Medicine - University of Pennsylvania, Philadelphia, USA.

${ }^{7}$ Bristol Urological Institute, Southmead Hospital, Bristol, United Kingdom.

Corresponding author. François Hervé (ORCID 0000-0002-9079-251X)

Urology department, Ghent University Hospital

De Pintelaan 185, 9000 Ghent, Belgium.

E-mail adress: francois.herve@uzgent.be

Telephone number: 0032 (0) 93321351

Fax number: 0032 (0) 93323889

WORDCOUNT: 5893 
Abstract

Aims: Nocturia, or waking up at night to void, is a highly prevalent and bothersome symptom. Currently, there is a lack of clear and consistent recommendations regarding evaluation and management of nocturia. The aim of this report is to discuss how to fill the gaps in our knowledge in order to develop a practical patient-oriented diagnostic and therapeutic algorithm for nocturia.

Methods: This paper is a report of the presentations and subsequent discussion of a Think Tank session at the annual International Consultation on Incontinence Research Society (ICI-RS) in June 2017 in Bristol.

Results and conclusion: Further investigations are needed to better understand the pathophysiology of nocturia, to allow improvement in diagnosis, and to optimize treatment by increasing efficacy and reducing adverse events. Patient-oriented practical guidelines on nocturia are needed to help clinicians from different disciplines diagnose and treat nocturia.

KEYWORDS: Nocturia, Nocturnal Polyuria, Pathophysiology, Diagnostics, Treatments, Practice Guidelines.

\section{INTRODUCTION}

Nocturia, or waking up at least one time at night to urinate, is a frequent and bothersome condition leading patients to seek assistance from specialists. Its prevalence increases with age and the presence of nocturnal voids $\geq 2$ times/night is reported in up to $60 \%$ of people older than 60 years ${ }^{1}$. It has been proven that the quality of life of patients with nocturia is impaired through a decline in both mental and physical health ${ }^{2}$. Moreover, patients with nocturia present a higher mortality rate, not only through an increased risk of falls and fractures ${ }^{3}$ but also through early death (OR=1.98) and cardiovascular disease $(\mathrm{OR}=4)^{4}$. The management of such a bothersome condition is an important issue and a matter of public health.

Nocturia is multifactorial and may be caused by nocturnal polyuria (NP), 24 hour (global) polyuria $^{5}$ (defined by the ICS as a production of more than $2.8 \mathrm{~L}$ of urine in 24 hours in adults), reduced voided volume (RVV), or a combination of the aforementioned issues ${ }^{2}$. However, this is a narrow "urological" view of nocturia. For each mechanism involved, a large number of underlying causative diseases can be held responsible. In a real-life 
situation, the symptom of nocturia is also diagnosed and treated by non-urological departments/disciplines such as general practitioners, geriatrics, cardiology, general internal medicine, gynaecology, neurology, rehabilitation, dietician and the sleep clinic, all of whom have their own guidelines and algorithms. Each discipline uses its own diagnostic and therapeutic pathways, and sometimes the symptom of nocturia is the primary target, other times it is a secondary target of the instigated therapies. Summarizing the current approaches from each of these disciplines is the first step towards a practical multidisciplinary approach to nocturia, and towards helping each of the disciplines learn how they could improve their approach just by visualizing it in a broader context. Identifying the current habits of the different disciplines through diagnostic and therapeutic packages based on an approach focusing on aetiology is a logical step as these packages are supported by validation and evidence within the disciplines. The next step will be to improve levels of evidence within the different packages of each specialty, and optimize the concept as a whole. These two last actions are not the aim of the current work. Another advantage of these causal, diagnostic and therapeutic packages is that identification of the different items involved in the different perspectives enhances recognition and enables more targeted action.

Historically, formal evaluation of nocturia has been compartmentalized into medical specialties as this symptom is likely to be encountered in a diverse range of clinical contexts. The multifactorial and multidisciplinary nature of nocturia is illustrated in Figure 1, the cornerstone of this article. Nocturia is here presented surrounded by 5 different causative/aetiological packages which are addressed when evaluating (diagnostic package) nocturia and when treating it (treatment package) by the respective disciplines. By demonstrating the current approach we hope to take the first step towards a more holistic approach.

The aim of the International Consultation on Incontinence-Research Society (ICI-RS) Nocturia Think Tank session in June 2017 was to identify the gaps in our knowledge and discuss what research is needed in order to develop a practical patient-oriented diagnostic and therapeutic algorithm for nocturia; the aim was therefore not to produce an algorithm at this time.

\section{METHODS}

The aim of the ICI-RS think tanks is to produce suggestions on future research based on lectures and discussion by experts (co-authors) in the field (http://www.ici-rs.org/). Retrospective studies, small pilots, and case reports can be used to strengthen the case for a hypothesis and future studies. The levels of evidence used in this sounding board are those from the European Association of Urology guidelines (http://uroweb.org/guidelines/). Conclusions on levels of evidence are based on published systematic reviews, meta-analyses and existing guidelines. Where this type of evidence is not available, evidence presented by the speakers is used and this is clearly indicated and differentiated from the robust evidence cited. This sounding board is not based on a systematic review performed by the panel. Indeed, such a review should be the next step, and represents an example of further research that is needed.

\section{DIAGNOSIS OF NOCTURIA BASED ON CAUSATIVE PACKAGES}


For each one of the different causative factors listed in the cornerstone figure of this article (Figure 1), some aspects of the diagnosis of nocturia have been discussed during the ICI-RS Nocturia Think Tank session, with the aim to discuss the gaps in our knowledge, and to give rise to ideas for further studies, with the ultimate, longer-term objective of building up an algorithm. It is clear that some causes can straddle several packages and that combinations of packages are often encountered in real-life situations.

\section{Urological package:}

Diagnosis (and management) of patients with nocturia involves completion of a series of bladder diaries in order to assess nocturia and to diagnose nocturnal polyuria (NP). For the paraclinical evaluation of urological factors which can lead to nocturia, special attention has to be paid to the lower urinary tract. Bladder, prostate and urethra have to be addressed as they are directly anatomically involved in the normal process of micturition.

Nocturnal post-void residual (PVR), a specific nocturia population:

Intra-individual variation of post-void residual is high and depends on the time of day and on the pre-void volume. Decalf et al. ${ }^{6}$ demonstrated that residual urine in an older population with nocturia is higher during the night than during the day.

From a clinical point of view, it may be important to identify patients who have increased nocturnal post-void residual. In doing so, one can specifically address nocturnal lower urinary tracts symptoms (LUTS), in the patient's work up and in developing treatment strategies.

Based on cases observed at our outpatient clinic or during hospitalization, there are different possible explanations for this phenomenon and the following possible underlying mechanisms have been hypothesized:

1) Hypothesis 1: NP associated with the loss of bladder sensation:

PVR and urinary retention can be a sign of the loss of bladder sensation. When combined with NP, it may contribute to over-distention of the bladder in older patients.

2) Hypothesis 2: NP associated with bladder outlet obstruction (BOO): Anatomical or functional BOO is a common cause of PVR. Insufficient contractility due to overfilling of the bladder with a high nocturnal diuresis rate in patients with BOO can potentially lead to a higher risk of nocturnal urinary retention.

3) Hypothesis 3: NP associated with detrusor underactivity:

The supplementary effect of NP on detrusor underactivity increases the risk of retention.

Abnormal filling and/or emptying of the bladder can be linked to intermittent overdistension of the bladder, especially in older patients with NP.

Based on observations from case reports, patients with combined NP and LUT dysfunction (loss of sensation, B00, detrusor underactivity) could be considered as a specific nocturia population.

Proposed research studies: 
- Further studies should focus on patients with nocturia due to NP rather than those with awakenings without NP.

- A lot of studies include patients with a limited number of nightly voids, which sometimes does not reflect the reality seen at the outpatient clinic and may allow a large placebo effect. On the other hand, when one selects patients with 3 awakenings or more, one ends up with a multifactorial disorder rather than a group with a single cause, and this might affect efficacy of the selected treatment in another way. Future studies should include patients with higher nocturia frequency.

- Delineate and study subgroups of patients with genuine lower urinary tract dysfunction and nocturnal polyuria through studying micturition-residual urine charts (a combination of a bladder diary and post-void residual records) in combination with daytime urodynamics.

Relationship of diuresis and urge perception in nocturia:

Locked within each of the urological factors are parameters such as volume per void and the perception of the degree of desire to void. It has previously been shown that there is a relationship between voided volume and the desire to void ${ }^{7}$. Does it matter what the rate of urine production might be in enhancing the perception of need to void, and is day different than night in this regard? Weiss et al. (unpublished data) have retrospectively analyzed voiding diaries of men and women with a chief complaint of LUTS presenting to an ambulatory urology clinic. Each voided volume was divided by the time elapsed from the previous void, yielding the diuresis rate (DR). Spearman's rank correlation coefficient rho was utilized to relate DR to the urgency perception score (UPS, with a graded response from $0-4$ where 0 is zero desire to void and 4 is a severe urgency). Correlations of DR, UPS and nocturia severity (ANV, actual number of nightly voids) were then calculated for both the entire cohort and individual subjects. Two hundred and two diaries from 136 unique patients (110 men, 26 women, mean age 65 years) were analyzed. The mean number of voids was 10.2 per diary. Results, depicted in Table 1, were that nocturnal DR correlated with nocturia severity but not with nocturnal UPS. Figure 2 is a histogram showing the frequency of different Spearman coefficients among the group. Below each bar there is a "bin" showing the range of coefficients, and above it is the number and percentage of patients in that category. There are two conclusions to be drawn from this graph. First, the relationship between DR and UPS is not a strong direct one for all patients. Second, $19 \%$ of subjects had a moderate correlation (rho 0.30.5 ) and $17 \%$, a strong correlation (rho above 0.5 ), indicating that $36 \%$ of the patients demonstrated a relationship between DR and the degree of desire to void.

Therefore, while nocturnal DR was moderately correlated with nocturia severity, the degree of correlation between DR and UPS varies significantly between individuals. Intuitively we might expect a stronger, more direct relationship between DR and UPS, further challenging our understanding of the sensory stimuli that promote the desire to void and supporting the notion that urgency is multifactorial. It would appear that measurement of DR in patients with nocturia may identify a cohort for whom influencing the rate of urine production may benefit the symptom.

Figure 3a depicts an individual who has no correlation between DR and UPS (rho=0.09) while Figure 3b depicts an individual with a strong correlation (rho=0.65). 
In order to assess micturition frequency, bladder capacity, instead of using handwritten data by patients, it would appear to be beneficial for them and for caregivers to directly record diary data into a computerized smartphone app which can then be uploaded to a secure website allowing for automated nocturia parameter calculations and graphical display of serial diary/questionnaire comparisons.

Proposed research studies:

- What are the differences in DR (both diurnal and nocturnal) between patients with and without nocturia?

- How do nocturia patients with a strong correlation between DR and UPS respond to behavioral modification therapies (alteration of intake, mid-day leg elevation, daytime use of lower extremity compression stockings, urge suppression techniques)

- What are the mechanisms that lead to some patients experiencing urgency, regardless of volume voided and DR?

- $\quad$ To use automated bladder diaries to assist in clinical research.

\section{Nephrological package:}

In order to determine the cause of nocturia and to discriminate between water and solute diuresis as pathophysiological mechanisms underlying nocturnal polyuria, a collection of 8 urine samples over $24 \mathrm{~h}$, with an interval of $3 \mathrm{~h}$ between each urine sample, can be used (renal function profile) ${ }^{8}$.

Clinical phenotyping may be a good suggestion to understand the underlying pathophysiological mechanisms and could then help clinicians to propose the most effective and safe treatment. Increasing salt clearance is found in spinal cord injury patients, hormonal deficiency and the elderly ${ }^{9}$. Renal function profiles could be reserved for complicated cases, therapy-resistant patients or for scientific purposes.

Finally, nephrogenic diabetes insipidus, hypercalciuria or polyuria due to renal disease can cause nocturia. Hypercalciuria is related to osmolar excretion in children with nocturnal enuresis ${ }^{10}$.

\section{Hormonal package:}

Hormonal secretion and changes in sex hormone levels due to menopause in women and to andropause in men have an impact on sodium excretion/reabsorption (through the renin-angiotensin-aldosterone system) and on water excretion (through antidiuretic hormone, arginine vasopressin [AVP]). We refer to the ICI-RS article from 2014 in relation to this subject ${ }^{11}$.

\section{Sleep package:}

Sleep deprivation leads to changes in the normal rise in AVP at the beginning of the night, especially in men, and leads to a nighttime increase in urine production ${ }^{12}$. Nevertheless, this evidence is a matter of debate as the study of Duffy et al. ${ }^{13}$ contradicts these findings, showing that 24 hours sleep deprivation in a carefully conducted laboratory protocol controlling posture, and hourly aliquoted fluid and food intake, did not 
result in an increase in urine production.Sleep apnea patients have impaired dipping and nighttime natriuresis mostly related to an increased plasma level of atrial natriuretic peptide (ANP). This increase in ANP is predominantly elicited by hypoxia during the apnea episodes, but is also related to release of ANP by the mechanical exposure of the atria. Restless legs are associated with nocturnal polyuria ${ }^{14}$.

Diagnosis is suggested clinically or through validated sleep questionnaires (e.g. Berlin sleep questionnaire, Pittsburg Sleep Quality Index, Epworth Sleepiness Scale) and can be confirmed by polysomnography and actigraphy. Further nocturia symptom-specific questionnaires to study the impact of nocturia on sleep include the Nocturia Impact Diary and the Nocturia Quality of Life Questionnaire.

Proposed research studies:

- Variables such as duration of first undisturbed sleep period (FUSP), sleep quality, insomnia and their importance with respect to nocturia frequency, should be discussed in future studies.

We also refer to the Nocturia Think Tank held during the ICI-RS 2015 concerning sleep issues in nocturia patients ${ }^{15}$.

\section{Cardiovascular package:}

Nocturia can be caused by an increase in diuresis following an increase in volume of the central vascular system (increase in end-diastolic/systolic volume or pressure). This can be screened clinically on physical examination (edema, varices) and by measuring the blood pressure.

Patients with lower limb venous insufficiency and congestive heart failure can experience water accumulation during the day, which is evacuated when lying down at night, causing NP.

Elevated blood pressure during the night is also a cause of nocturia. It has been shown that a pronounced dipping is accompanied by reduced urine production and that attenuated dipping leads to increased urine production. This is seen in the polyuric type of monosymptomatic bedwetting, not as a constant phenomenon but corresponding with the incidence of bedwetting ${ }^{16}$. Based on the recent findings of the $108^{\text {th }}$ Nobel prize $^{17}$, altered circadian rhythmicity on a subcellular level (molecular mechanisms) in cardiac and renal tissues might also explain such a change in 24-hour blood pressure and nocturnal polyuria.

In elderly men with LUTS, it has been shown that nighttime polyuria, only present in some of the patients, was related to a late evening natriuresis which persisted through the night, a phenomenon which was not present in non-symptomatic males and males with RVV day and night ${ }^{18}$. Patients with spinal cord injury seem to have lost their nighttime blood pressure dipping, especially with high lesions ${ }^{9}$.

Proposed research studies: 


- Study the relationship between 24-hour blood pressure
measurements and nocturia.

\section{Intake package:}

Fluid, salt and food intake, as well as diabetes and obesity, may increase diuresis rate and may alter the circadian rhythm of urine production. During ICI-RS 2014, the Nocturia Think Tank session explored the link between LUTS and metabolic disorders ${ }^{19}$. Intake diaries can be useful but linking diuresis information to intake of salt, protein, calcium, potassium and so on is the work of a dietician. Renal function profiles can also help to estimate the intake of these substances ${ }^{8}$.

\section{Nocturia screening tool: TANGO (Targeting the individual's Aetiology of Nocturia to Guide Outcomes):}

To date a clear and sequential databank to capture the key information from the different causative factors and their diagnostic tools (bladder diaries, sleep measures, results of renal function tests and urine flow dynamics etc.) has not been developed. Guidance about interpreting findings is largely drawn from clinical experience; an interpretation cascade has not been formalized. There is also a need to simplify the interpretation process so that it can be generalized across specialties and service models.

A brief patient-completed screening tool has been developed in order to capture symptoms associated with the full breadth of causes of nocturia: TANGO20. The final TANGO Short Form (TANGO SF) (hitherto known as TANGO) comprised 22 items that fell into four domains. The Cardiothoracic domain captured the presence of disorders that contribute to 24 hour polyuria and NP. The Sleep domain classified an individual's quality of sleep, quantified the initial sleep period before first awakening to void, identified causal factors for waking in the night aside from nocturia and identified likely sleep disordered breathing. The Urinary tract domain facilitates the identification of LUTS of clinical relevance to nocturia. The final domain related to Wellbeing, specifically poor health, the occurrence of falls, excessive daytime sleepiness and loss of enthusiasm.

A number of clinical contexts are gender-specific, raising the possibility that TANGO could be segregated to better meet the service needs. Review of recent literature and sub-group analysis of the original TANGO Long form data has led us to propose several additional gender-specific items. For TANGO-Male it has been proposed to add items relating to low testosterone levels: waking because of feeling hot or sweating, waking because of feeling cold and not having had a blood test to verify testosterone levels. TANGO-Female would lose the question related to BOO and include items about symptoms of estrogen depletion and menopausal status.

Proposed research studies:

- Extend the number of languages in which TANGO is available.

- Additional multi-center concurrent validation of TANGO will complete psychometric testing. Investigation of performance of gender-specific items.

TREATMENT OF NOCTURIA BASED ON CAUSATIVE PACKAGES 
For each one of the different causative factors listed on the cornerstone figure of this article (Figure 1), some aspects of the treatment of nocturia have been discussed during the ICI-RS Nocturia Think Tank session, with the aim of identifying the gaps in our knowledge, and generating ideas for further studies, looking to develop an algorithm as the ultimate goal. It is true that different packages are merged already on a regular basis as colleagues refer to each other's pathways.

\section{Urological package:}

Treating reduced voided volume (RVV)

RVV (global or nocturnal) can be caused by B00, detrusor underactivity and/or overactive bladder (OAB).

B00, such as that caused by benign prostatic enlargement (BPE), may cause storage and/or voiding symptoms. Medications targeting these disorders have been tested in the treatment of nocturia. Alpha blockers ${ }^{21-23}$ and 5- $\alpha$-reductase inhibitors ${ }^{21,24}$ or a combination of both ${ }^{5}$ are recommended by EAU guidelines (Level of Evidence $1 \mathrm{~b}$ for both, Grade B and C respectively) in male nocturia patients with LUTS (and enlarged prostate for 5 - $\alpha$-reductase inhibitors $)^{25,26}$.

For $\mathrm{OAB}$, a positive effect on nocturia has been shown with medical treatment, intradetrusor injections of Botox $^{27}$ and sacral neuromodulation ${ }^{28}$. Antimuscarinics are recommended by EAU guidelines in nocturia patients with OAB symptoms (Level of Evidence 1b, Grade B) ${ }^{25,26}$. In a network meta-analysis conducted by Buser et al. ${ }^{29}$, all studies showed that antimuscarinics improved nocturia episodes when compared to placebo. Brubaker et al. ${ }^{30}$ specifically looked at patients with nocturnal polyuria (NP) and did not find any statistical difference in the reduction of nocturia episodes in patients with NP treated by solifenacin. Next to anticholinergics, Mirabegron, a $\beta 3$ adrenoreceptor-agonist, offers a recent alternative treatment for overactive bladder with a reduction of nocturia episodes and low occurrence of side effects ${ }^{31}$.

Some authors also pointed out a statistically significant reduction of nocturia in patients treated with OnabotulinumtoxinA injected in the detrusor, for example in the study of Nitti and al. ${ }^{27}$, with a reduction of 0.45 episodes of nocturia per day 12 weeks after the injection of 100 units of OnabotulinumtoxinA.

Sacral neuromodulation (SNM) is an electrical stimulation therapy which has been applied for decades in various conditions including urinary urge incontinence due to idiopathic detrusor overactivity on one hand, and urinary retention on the other hand. Powell et al. ${ }^{32}$ studied the effect of SNM on 82 patients (72 women, and 10 men) and showed a reduction in nocturia episodes with less benefit for patients older than 70 years old and for those with $<3$ preoperative nocturia episodes.

Combination treatments of $\alpha$-blockers and antimuscarinics, and desmopressin and antimuscarinics, are currently being tested in the treatment of nocturia ${ }^{33}$.

Nephrological package:

\section{Antidiuretic therapy}


Antidiuretic therapy for NP can be offered using AVP analogues, which specifically bind to V2-receptors in the kidneys and thereby increase water permeability, enhance water reabsorption and hence dilute extracellular fluid ${ }^{34}$. This leads to concentration of urine and a decrease in urine output. The EAU 2017 guidelines recommend desmopressin (peptide V2-receptor agonist) to treat NP in men under the age of 65 (Level of Evidence $1 \mathrm{a}$, Grade A recommendation) ${ }^{25}$. Due to the possible severe side-effect of hyponatremia, screening at baseline, during dose titration and during treatment is recommended ${ }^{25}$. A low dose gender-specific orally disintegrating tablet formulation is available in Europe ${ }^{35,36}$ and a low dose intranasal spray formulation in the US ${ }^{37}$, and both have been demonstrating an improved safety profile making the therapy accessible for older people. These ideas of low dosing and gender specificity fit well in a holistic approach increasing safety and efficacy through a patient-oriented approach, but of course the effectiveness of this concept in the clinic will need to be monitored in the future. New AVP analogues are currently being developed, allowing further dose reduction to achieve a lower risk of hyponatremia and possible application in older patients.

The combined use of desmopressin with furosemide with an appropriate timing have also to be taken into account in our treatment armamentarium in patients with resistant nocturnal polyuria based on its multiple pathogenic factors, as addressed in the pedriatic study of De Guchtenaere ${ }^{38}$, but also in a randomized placebo controlled trial of a staggered furosemide and desmopressin by $\mathrm{Fu}$ et $\mathrm{al} .{ }^{39}$.

Proposed research studies:

- Trial of timed diuretic therapy in men with nocturia due to NP (as advised by the EAU Guidelines (Level of Evidence 1b, Grade C recommendation) ${ }^{25}$.

- Evaluation of combination therapy using diuretics and V2-receptor specific AVP analogues, allowing the mechanisms of action to complement each other.

- The mechanism of action of the V2-receptor involves the aquaporin 2 (AQP2) channel. Transcriptional upregulation, or drugs activating or potentiating these channels, could also improve both 24 hour polyuria and NP.

\section{Targeting both NP and RVV}

While most of the studies focus on the number of voids, some articles have recently shown that both alpha blockers ${ }^{40,41}$ and anticholinergics ${ }^{42}$ are effective in decreasing nocturnal urine volume. Another study by Choi et al. ${ }^{43}$ concluded that NP decreased when relieving LUT obstruction using prostatic laser enucleation. We may wonder to what extent there is a communication between LUTS and the kidneys, i.e. the way urine is produced. This could be a new strategy for treating NP.

Prostaglandin E2 inhibits the action of AVP in the collecting duct and can increase detrusor tone and enhance micturition. Cyclo-oxygenase (COX) inhibitors celecoxib ${ }^{44}$ and loxoprofen sodium ${ }^{45}$ have been tested and Paxerol ${ }^{\mathrm{TM}}$ (Acetaminophen and Ibuprofen combination) is currently in phase II trials for nocturia.

Proposed research studies:

- Further evaluation of prostaglandin E2 inhibition in the treatment of NP and RVV 
- A combination of COX-inhibitors and desmopressin may be worth investigating as they complement each other's action at the kidney level.

- Another pathway worth considering is the role of hypercalciuria in nocturia as this has been shown to interfere with antidiuretic hormone and related aquaporin $2^{46}$ as well as reduce functional bladder capacity and induce overactive bladder ${ }^{47}$ Should hypercalciuria be present in nocturia patients, it may be worth treating, or it may be worth aiming to reduce nocturnal calcium levels in the urine to further complement the action of other medication used in the treatment of nocturia.

- To study the change in urine production after LUTS medication or surgeries.

- To evaluate the communication between lower urinary tract and kidneys.

Treatment of NP in relation to its causality (water versus salt diuresis)

In the case of NP, one can hypothesise that NP caused by water diuresis is best treated with desmopressin, and NP caused by increased salt diuresis is best treated by saltreducing diuretics taken during the day ${ }^{48}$. In case of combined causality, combination therapy can be suggested; however these therapeutic hypotheses have never been validated in a randomised controlled trial (RCT). A consortium of doctors from the international NP research group suggested in 2016 a trial design, summarized in Figure 4, in which all patient with nocturia are eligible (not based on any type of pathogenic factor for nocturia).

\section{Hyponatremia as a complication of medical treatment}

Until recently hyponatremia occurred in around 5\% of the RCT patients ${ }^{49,39}$. Accordingly, precautions to detect this complication were suggested. The main factors were advanced age, low baseline sodium levels, glomerular filtration rate (GFR) below $60 \mathrm{ml} / \mathrm{min}$, important leg edema and heart failure. Juul et al. $^{50}$ found that there is a gender dependent variable sensitivity to desmopressin, resulting in the commercialisation of a low dose and gender-specific formulation of desmopressin with an improved safety profile as a result.

These preclinical findings of low-dose and gender specific formulation have been clinically confirmed in two trials ${ }^{35,36}$. The low-dose safety has been confirmed in a conference abstract from Kaminetsky et al. using a nasal spray formulation ${ }^{51}$, but not the gender. Larger comparative pharmacokinetic and pharmacodynamics studies are needed to explain the difference in findings.

In Juul's article ${ }^{50}$, a sodium monitoring plan is proposed whereby baseline sodium must be $\geq 135 \mathrm{mmol} / \mathrm{L}$ (especially important in the elderly), with additional monitoring at week 1 and month 1 for those at elevated risk because they are aged $\geq 65$ years or receiving concomitant medication associated with hyponatremia.

From the studies using this low dose and gender-specific formulation, a decreased hazard ratio for the development of hyponatremia was found for some patients with the following factors: low baseline sodium, decreasing GFR, increasing age and high dose of the drug. A number of other factors are also suggested in the literature (hemoglobin, hematocrit, concomitant medication, water- or salt- reducing NP, heart failure and leg edema) and merit further attention.

\section{1) Hemoglobin, hematocrit and hyponatremia}


Hemoglobin and hematocrit have been reported to be related to hyponatremia in general and authors hypothesize inflammation as a potential mechanism. Haptoglobin subtyping might be another plausible explanation for this phenomenon.

Patients with subtype Hp1-1 have a greater risk for the presence of 24 hour polyuria. The higher diurnal diuresis level may be explained by a diminished sodium clearance, which is consistent with the finding that Hp1-1 is related to salt-sensitivity and hypertension because of the possible increase in intravascular volume by accumulation of sodium and water. Patients with Hp2-2 subtype are known to be salt-resistant, which is not the case in patients with $\mathrm{Hp} 1-1^{52}$.

Proposed research studies:

- Link haptoglobin subtypes and salt handling with hemoglobin and hematocrit.

- Study why these haptoglobin subtypes have different salt handling, investigating kidney, blood/plasma, interstitium, intracellular mechanisms.

- Investigate the relationship between haptoglobin subtype and risk of hyponatremia after desmopressin treatment.

\section{2) Concomitant medication and hyponatremia}

Many drugs have been related to hyponatremia in the literature. We would like to focus on medications that are related to an increased loss of sodium (i.e. salt-reducing diuretics, ACE inhibitors, angiotensin II receptor blockers). It is logical to hypothesize that combining drugs that cause hyponatremia will increase the risk of its occurrence but this has never been studied. As most older people use multiple medications, more research is needed in this field. Probably, it is not only the fact of combining these medications but also their mechanism of action, timing, formulation (immediate release versus extended release) and half-life of the drug that are important. Indeed, the salt gradient around the loop of Henle is more likely to develop a steady state, with resetting of the gradient, with drugs with a long half-life than with drugs with a short half-life. Timing might be extremely important, so desmopressin (nocturnal water retention) in combination with a short-acting (salt- and water-reducing) diuretic (e.g. furosemide) during the day may be safer than combining both drugs in the evening.

Proposed research studies:-

- $\quad$ Predicting the risk of hyponatremia is a major issue: there is a need for larger datasets to explore the risk of hyponatremia, particularly in an older population with many comorbidities and concomitant medications.

3) Heart failure, leg edema and hyponatremia

Heart failure induces atrial dilatation resulting in high ANP and in salt and water loss. Leg edema without heart failure results in nocturnal atrial dilatation, high nocturnal ANP and salt and water loss during the night. Adding desmopressin in the late evening means adding nocturnal water retention to the salt loss, causing additive risk for development of hyponatremia.

Proposed research studies:

- Investigation of how to screen for heart failure and estimate the concomitant risk of hyponatremia

- $\quad$ Explore to what extent leg edema is a risk for hyponatremia 
- $\quad$ To study the effect of leg elevation in order to understand the contribution of first line treatment for peripheral edema

- Can combined therapy of leg edema plus desmopressin be a solution to decrease the risk and enhance the benefit?

\section{Hormonal package:}

These therapies have not been addressed in the EAU guidelines.

The net effect of all sex hormone is antidiuretic, which might indicate a reason for postmenopausal hormone substitution in patients with bothersome nocturia. We refer to the ICI-RS article from 2014 in relation to this subject ${ }^{11}$.

\section{Sleep package:}

Medication improving sleep has been tested in nocturia. Sedatives are recommended by the EAU (Level of Evidence 2, Grade C) ${ }^{25}$ to help patients with nocturia return to sleep. Melatonin has also been shown to have a beneficial effect on nocturia ${ }^{53}$.

The efficacy of continuous positive airway pressure therapy (CPAP) had been shown in studies by Miyauchi ${ }^{54}$ and Miyazato ${ }^{55}$ with decrease of nocturia episodes and nocturnal urine production as outcome.

Proposed research studies:

To evaluate the presence of, role of, and possible interventions affecting clock genes as a new and promising pathway in nocturia treatment

We also refer to the Nocturia Think Tank held during the ICI-RS 2015 concerning sleep issues in nocturia patients ${ }^{15}$. This report stresses out the need to treat sleep disorders such as obstructive sleep apnea and periodic limb movements during sleep and to consider dopaminergic neurotransmission as a potential mechanism involved which may be a target for treatment (like dopamine-agonist in Parkinson disease).

\section{Cardiovascular package:}

Diuretic treatment is part of the medical armamentarium in cardiovascular disorders and optimal timing of the diuretic can aid in preventing symptoms of nocturia. Furosemide 40mg taken 6 hours before sleep (mid-afternoon) has been shown to improve nocturia ${ }^{56}$.

In the treatment of nighttime polyuria little attention has been paid to the lowering of blood pressure and its impact on nocturnal urine volume. Aside from improvements in sleep which might lead to a better dipping profile, there might perhaps be a role for short-acting antihypertensives.

Proposed research studies:

- Study short-acting antihypertensives, with the aim of inducing a reduction of blood pressure during the night in patients with non-dipping hypertension.

\section{Intake package:}


When underlying medical conditions (e.g. congestive heart failure, bladder calculi, primary polydipsia, obstructive sleep apnea, renal failure) have been excluded as a cause of nocturia or have been treated by the appropriate medical expert, some conservative measures can be offered, out of the "kitchen sink". These consist of behavioral interventions (e.g. cessation of fluid intake 4 hours before bedtime, reducing caffeine and alcohol, improving sleep hygiene; Level of Evidence 4, Grade A). Additional interventions (e.g. compression stockings for edema, changing or stopping drugs which may cause nocturia) can be proposed. This structured multimodal approach has been studied by Vaughan et $\mathrm{al}^{57}$.

Recently, a Japanese non-RCT but controlled study demonstrated that after reducing salt intake, patients had a significant decrease in nocturia episodes per night (from $2.3 \pm 0.9$ to $1.4 \pm 1.0$ ) and a significant improvement in quality of life parameters (from $3.6 \pm 1.2$ points to $2.7 \pm 1.3$ points) ${ }^{58}$. This work was presented as an abstract and a full paper has not yet been published. Further studies in this field are needed.

\section{FUTURE RESEARCH}

\section{Focus on non-pharmacological, non-surgical treatments:}

There is currently little consensus about the impact of individual first-line therapies for reducing nocturia episodes. The efficacy of individual changes in behavior or lifestyle, particularly after sub-classification by presence or absence of nocturnal polyuria, is unknown. A systematic evaluation of the existing evidence from randomised and controlled trials is therefore needed to determine effectiveness and safety of these firstline modalities. We ran an initial search in Medline for such studies. From 760 possible studies, 46 were retained (see Appendix for the list of the studies). On the basis of design there were 17 RCTs describing 2741 participants; 6 controlled trials with a total of 257 participants and 17 cohort studies describing 846 participants. Placebo arm data was available for 2546 participants from 6 studies. Nocturia episodes were reported in 24 studies and change in nocturia episodes in 22 studies. Change in FUSP was described in 2 studies. Nocturia was the primary and secondary measure in 37 and 7 studies respectively. Identification of these studies suggests that a formal systematic review is warranted to evaluate the evidence base for the effectiveness of individual and combined behavioral therapies in reducing nocturia episodes. Findings will guide clinicians when making clinical decisions.

\section{Focus on treatment packages based on causative factors:}

Rather than just studying certain "department-oriented treatments", we also need to evaluate the impact of "patient-oriented therapy packages" based on causative factors as described above. An overview of research gaps/future research is presented in Figure 5 and the current knowledge concerning behavioural and medical therapies available to treat nocturia is summarized in Figure 6. This figure has been designed to show that the levels of evidence of our treatment armamentarium have to be improved, and to point out research gaps. The pyramid has been created in light of the EAU Male LUTS guidelines ${ }^{25}$ and of a review of individual ICI-RS speakers' presentations. This is by no means a figure of recommendations and we stress that the management of nocturia must be individually tailored.

Further studies will aim to:

- Improve the level of evidence for the already tested treatments. 
- Introduce some other therapies not listed in the segmented pyramid, and try to bring them to the top of the pyramid.

\section{Ideas of future directions on nocturia research (from the perspective of a journal editor):}

In order to optimize reporting related to nocturia and interventions for the condition, journal requirements should include a standardization of expected outcomes and populations undergoing interventions. The need to clearly define not only the nocturnal frequency associated with nocturia burden but also coexistent NP is critical to adequately define the population undergoing intervention.

Furthermore, the importance of using standardized outcomes will be critical in providing consistent and comparable data sources for reporting purposes. Standardization should include reproducible methods for capturing nocturia episodes and NP, generally accepted definitions for NP volumes, and the use of standardized quality of life outcome measures. The recent development of a fully-validated instrument should be used as a metric for subsequent development of combined subjective and objective measures.

The use of meta-analytic techniques should be minimized given the complexity of historical data reporting in this area and additionally the apparent overuse of such methods.

\section{CONCLUSION}

Nocturia is a frequent and bothersome condition and an important issue of public health. Unfortunately, when managing such a multifactorial symptom, specialists have to deal with the limited knowledge concerning nocturia, and they may remain isolated with insufficient interaction with other specialties. Specialists therefore face a lack of consistent recommendations and patient-oriented diagnosis and treatment algorithms.

Further work is needed to fully understand underlying mechanisms leading to nocturia, to diagnose it correctly and easily, and to improve treatments while ensuring low adverse event rates.

A multidisciplinary approach is the cornerstone of the management of nocturia. Partnership between specialties should be promoted and more patient-specific therapy needs to be developed.

The International Consultation on Incontinence-Research Society (ICI-RS) Nocturia Think Tank therefore stresses the importance of developing practical patient-oriented guidelines for nocturia.

\section{LEGENDS}

Figure 1: $\quad$ Multifactorial and multidisciplinary character of nocturia.

Table 1: Spearman's rank correlations between diuresis rate, urge perception score and nocturia severity. 
Figure 2: $\quad$ Histogram showing distribution of Spearman correlation coefficients across the entire cohort.

Figure 3a: Patient lacking correlation between DR and UPS.

Figure 3b: Patient with strong correlation between DR and UPS.

\author{
Figure 4: Potential RCT design to demonstrate a more etiologically-driven \\ treatment of NP.
}

Figure 5: $\quad$ Overview of research gaps/future research.

Figure 6: $\quad$ Segmented pyramid showing the current knowledge concerning behavioral and medical therapies available to treat nocturia

\title{
This is by no means a figure of recommendations and we stress that the management of nocturia must be individually tailored. LoE= Level of evidence \\ References
}

1. Bosch JLHR, Weiss JP. The Prevalence and Causes of Nocturia. JURO. 2013;189:S86-S92.

2. Oelke M, Adler E, Marschall-Kehrel D, Herrmann TRW, Berges R. Nocturia: state of the art and critical analysis of current assessment and treatment strategies. World J Urol. 2014;32(5):1109-1117.

3. Temml C, Ponholzer A, Gutjahr G, Berger I, Marszalek M, Madersbacher S. Nocturia is an age-independent risk factor for hip-fractures in men. Neurourol Urodyn. 2009;28(8):949-52.

4. Kupelian V, Fitzgerald MP, Kaplan SA, Norgaard JP, Chiu GR, Rosen RC. Association of nocturia and mortality: results from the Third National Health and Nutrition Examination Survey. J Urol. 2011;185(2):571-7.

5. Abrams P, Cardozo L, Fall M, et al. The standardisation of terminology in lower urinary tract function: Report from the standardisation sub-committee of the International Continence Society. Urology. 2003;61(1):37-49.

6. Decalf V, Huion A, Denys M-A, Kumps C, Petrovic M, Everaert K. Circadian Variation in Post Void Residual in Nursing Home Residents With Moderate Impairment in Activities of Daily Living. J Am Med Dir Assoc. 2017;18(5):433-437.

7. Blaivas JG, Tsui JF, Amirian M, et al. Relationship between voided volume and the urge to void among patients with lower urinary tract symptoms. Scand J Urol. 2014;48(6):554-558.

8. Nocturnal Polyuria: Excess of Nocturnal Urine Production, Excess of Definitions-Influence on Renal Function Profile. J Urol. 2016;195(3):670-676.

9. Goh MY, Millard MS, Wong ECK, Brown DJ, Frauman AG, O'Callaghan CJ. Diurnal blood pressure and urine production in acute spinal cord injury compared with controls. Spinal Cord. 2017;55(1):39-46.

10. Raes A, Dossche L, Hertegonne N, et al. Hypercalciuria is Related to Osmolar Excretion in Children With Nocturnal Enuresis.

11. Hanna-Mitchell AT, Robinson D, Cardozo L, Everaert K, Petkov G V. Do we need to know more about the effects of hormones on lower urinary tract dysfunction? ICI-RS 2014. Neurourol Urodyn. 2016;35(2):299-303.

12. Kamperis K, Hagstroem S, Radvanska E, Rittig S, Djurhuus JC. Excess diuresis and natriuresis during acute sleep deprivation in healthy adults. AJP Ren Physiol. 2010;299(2):F404-F411.

13. Duffy JF, Scheuermaier K, Loughlin KR. Age-Related Sleep Disruption and Reduction in the Circadian Rhythm of Urine Output: Contribution to Nocturia? Curr Aging Sci. 2016;9(1):34-43.

14. Van Kerrebroeck P, Abrams P, Chaikin D, et al. The standardization of terminology in nocturia: report from the standardization subcommittee of the International Continence Society. BJU Int. 2002;90 Suppl 3:11-5.

15. Denys M-A, Cherian J, Rahnama'i MS, et al. ICI-RS 2015-Is a better understanding of sleep the key in managing nocturia? Neurourol Urodyn. April 2016.

16. Kahraman A, Dursun H, Hatipoglu S, et al. Non-dipping phenomenon in children with monosymptomatic nocturnal enuresis. Pediatr Nephrol. 2013;28(7):1099-103.

17. Sehgal A. Physiology Flies with Time. Cell. 2017;171(6):1232-1235.

18. Matthiesen TB, Rittig S, Nørgaard JP, Pedersen EB, Djurhuus JC. Nocturnal polyuria and natriuresis in male patients with nocturia and lower urinary tract symptoms. J Urol. 1996;156(4):1292-9.

19. Denys M-A, Anding R, Tubaro A, Abrams P, Everaert K. Lower urinary tract symptoms and metabolic disorders: ICI-RS 2014. Neurourol Urodyn. 2016;35(2):278-282.

20. Bower WF, Rose GE, Ervin CF, Goldin J, Whishaw DM, Khan F. TANGO - a screening tool to identify comorbidities on the causal pathway of nocturia. BJU Int. 2017;119(6):933-941.

21. Johnson TM, Jones K, Williford WO, Kutner MH, Issa MM, Lepor H. CHANGES IN NOCTURIA FROM MEDICAL TREATMENT OF BENIGN PROSTATIC HYPERPLASIA: SECONDARY ANALYSIS OF THE DEPARTMENT OF VETERANS AFFAIRS COOPERATIVE STUDY TRIAL.

22. Ceylan C, Ceylan T, Doluoglu OG, Yuksel S, Agras K. Comparing the effectiveness of intranasal desmopressin and doxazosin in men with nocturia: a pilot randomized clinical trial. Urol J. 2013;10(3):993-8.

23. Simaioforidis V, Papatsoris AG, Chrisofos M, Chrisafis M, Koritsiadis S, Deliveliotis C. Tamsulosin versus transurethral resection of the prostate: Effect on nocturia as a result of benign prostatic hyperplasia. Int J Urol. 2011;18(3):243-248.

24. Oelke M, Roehrborn CG, D?Ancona C, Wilson TH, Castro R, Manyak M. Impact of dutasteride on nocturia in men with lower urinary tract symptoms suggestive of benign prostatic hyperplasia (LUTS/BPH): a pooled analysis of three phase III studies. 
Gratzke C, Bachmann A, Descazeaud A, et al. EAU Guidelines on the Assessment of Non-neurogenic Male Lower Urinary Tract Symptoms including Benign Prostatic Obstruction. Eur Urol. 2015;67(6):1099-1109.

26. Sakalis VI, Karavitakis M, Bedretdinova D, et al. Platinum Priority - Review - Voiding Dysfunction Medical Treatment of Nocturia in Men with Lower Urinary Tract Symptoms: Systematic Review by the European Association of Urology Guidelines Panel for Male Lower Urinary Tract Symptoms. 2017.

27. Nitti VW, Dmochowski R, Herschorn S, et al. OnabotulinumtoxinA for the Treatment of Patients with Overactive Bladder and Urinary Incontinence: Results of a Phase 3, Randomized, Placebo Controlled Trial. JURO. 2013;189:2186-2193.

28. Peters KM, Kandagatla P, Killinger KA, Wolfert C, Boura JA. Clinical outcomes of sacral neuromodulation in patients with neurologic conditions. Urology. 2013;81(4):738-743.

29. Buser N, Ivic S, Kessler TM, Kessels AGH, Bachmann LM. Efficacy and Adverse Events of Antimuscarinics for Treating Overactive Bladder: Network Meta-analyses. Eur Urol. 2012;62(6):1040-1060.

30. Brubaker L, FitzGerald MP. Nocturnal polyuria and nocturia relief in patients treated with solifenacin for overactive bladder symptoms. Int Urogynecol J Pelvic Floor Dysfunct. 2007;18(7):737-41.

31. Nitti VW, Auerbach S, Martin N, Calhoun A, Lee M, Herschorn S. Results of a randomized phase III trial of mirabegron in patients with overactive bladder. J Urol. 2013;189(4):1388-95.

32. Powell CR, Zabell J, Takacs EB, Kreder KJ. Reduction of Nocturia after Sacral Neuromodulation Therapy for Overactive Bladder. J Urol Res J Urol Res. 2015;2(1).

33. Nakanishi S. [Efficacy of mirabegron, a $\beta 3$-adrenergic agonist, switched from an anticholinergic agent for female patients aged over 70 years]. Hinyokika Kiyo. 2013;59(9):561-4.

34. Hammer M, Vilhardt H. Peroral treatment of diabetes insipidus with a polypeptide hormone analog, desmopressin. $J$ Pharmacol Exp Ther. 1985;234(3):754-60.

35. Weiss JP, Herschorn S, Albei CD, Van Der Meulen EA. Efficacy and Safety of Low Dose Desmopressin Orally Disintegrating Tablet in Men with Nocturia: Results of a Multicenter, Randomized, Double-Blind, Placebo Controlled, Parallel Group Study. JURO. 2013;190:965-972.

36. Sand PK, Dmochowski RR, Reddy J, Van Der Meulen EA. Efficacy and Safety of Low Dose Desmopressin Orally Disintegrating Tablet in Women with Nocturia: Results of a Multicenter, Randomized, Double-Blind, Placebo Controlled, Parallel Group Study. JURO. 2013;190:958-964.

37. NOCTIVA (desmopressin acetate) nasal spray.

38. De Guchtenaere A, Walle C Vande, Sintjan P Van, et al. Desmopressin Resistant Nocturnal Polyuria May Benefit From Furosemide Therapy Administered in the Morning.

39. Fu F-G, Lavery HJ, Wu D-L. Reducing nocturia in the elderly: A randomized placebo-controlled trial of staggered furosemide and desmopressin. Neurourol Urodyn. 2011;30(3):312-316.

40. Kojima Y, Sasaki S, Imura M, Kubota Y, Hayashi Y, Kohri K. Tamsulosin reduces nighttime urine production in benign prostatic hyperplasia patients with nocturnal polyuria: a prospective open-label long-term study using frequency-volume chart. Neurourol Urodyn. 2012;31(1):80-5.

41. Kim YW, Park J, Chung H, et al. The Effectiveness of Silodosin for Nocturnal Polyuria in Elderly Men With Benign Prostatic Hyperplasia : A Multicenter Study. 2015;19(2).

42. Yokoyama O, Homma Y, Yamaguchi O. Imidafenacin, an Antimuscarinic Agent, Improves Nocturia and Reduces Nocturnal Urine Volume. Urology. 2013;82(3):515-520.

43. Choi WS, Ku JH, Oh SJ, Kim SW, Paick JS. Change of nocturnal polyuria after holmium laser enucleation of the prostate in patients with benign prostatic hyperplasia. Urology. 2014;84(3):650-656.

44. Falahatkar S, Mokhtari G, Pourreza F, Alaeddin Asgari S, Noshad Kamran A. Prostatic Disease and Male Voiding Dysfunction Celecoxib for Treatment of Nocturia Caused by Benign Prostatic Hyperplasia: A Prospective, Randomized, Double-Blind, Placebo-Controlled Study.

45. Shin H Il, Kim BH, Chang HS, Park CH, Kim C Il. www.kjurology.org Long-Term Effect of Loxoprofen Sodium on Nocturia in Patients with Benign Prostatic Hyperplasia. Korean J Urol C Korean Urol Assoc Korean J Urol. 2011;52:265-268.

46. Procino G, Mastrofrancesco L, Tamma G, et al. Calcium-sensing receptor and aquaporin 2 interplay in hypercalciuriaassociated renal concentrating defect in humans. An in vivo and in vitro study. Dussaule J-C, ed. PLoS One. 2012;7(3):e33145.

47. Tomohiro M. Influence of urinary calcium excretion on urinary symptoms such as nocturia. Eur Urol Suppl. 2017;16(3):e977-e978.

48. Nørgaard JP, Hashim H, Malmberg L, Robinson D. Antidiuresis therapy: Mechanism of action and clinical implications Neurourol Urodyn. 2007;26(7):1008-1013.

49. van Kerrebroeck P, Rezapour M, Cortesse A, Th?roff J, Riis A, N?rgaard JP. Desmopressin in the Treatment of Nocturia: A Double-Blind, Placebo-Controlled Study. Eur Urol. 2007;52(1):221-229.

50. Juul KV, Malmberg A, van der Meulen E, Walle J Vande, Nørgaard JP. Low-dose desmopressin combined with serum sodium monitoring can prevent clinically significant hyponatraemia in patients treated for nocturia. BJU Int. 2017;119(5):776-784.

51. Kaminetsky J, Wein A, Dmochowski R, Herschkowitz S, Cheng M, Abrams S FS. A Randomized, Double-Blind, Placebo Controlled Study of 2 Doses of SER120 Nasal Spray in Patients with Nocturia. In: AUA Abstract. ; 2016:Moderated Poster MP74; Room 24.

52. Denys M-A, Goessaert A-S, Decalf V, et al. Haptoglobin polymorphism: an explanation for global polyuria? Neurourol Urodyn. 1982;34(suppl. 3):S28-S29.

53. Drake MJ, Mills IW, Noble JG. MELATONIN PHARMACOTHERAPY FOR NOCTURIA IN MEN WITH BENIGN PROSTATIC ENLARGEMENT.

54. Miyauchi Y, Okazoe H, Okujyo M, et al. Effect of the Continuous Positive Airway Pressure on the Nocturnal Urine Volume or Night-time Frequency in Patients With Obstructive Sleep Apnea Syndrome. Urology. 2015;85(2):333-336.

55. Miyazato M, Tohyama K, Touyama M, et al. Effect of continuous positive airway pressure on nocturnal urine production in patients with obstructive sleep apnea syndrome. Neurourol Urodyn. 2017;36(2):376-379.

56. Reynard JM, Cannon A, Yang Q, Abrams P. A novel therapy for nocturnal polyuria: a double-blind randomized trial of frusemide against placebo. Br J Urol. 1998;81(2):215-8.

57. Vaughan CP, Endeshaw Y, Nagamia Z, Ouslander JG, Johnson TM. A multicomponent behavioural and drug intervention for nocturia in elderly men: rationale and pilot results. BJU Int. 2009;104(1):69-74.

58. Tomohiro M, Nakamura Y, Yasuda T, Ohba K, Miyata Y, Sakai H. Effect of restricted salt intake on nocturia. Eur Urol Suppl. 
Table 1. Spearman's rank correlations between diuresis rate, urge perception score and nocturia severity.

\begin{tabular}{|c|c|c|}
\hline Diary Parameters & Spearman's $\rho$ & $\begin{array}{c}\text { Significance } \\
(\mathbf{p})\end{array}$ \\
\hline Nocturnal DR \& ANV & $\mathbf{0 . 4 2}$ & $<\mathbf{0 . 0 0 1}$ \\
\hline Diurnal DR \& ANV & -0.024 & 0.8209 \\
\hline Diurnal DR \& Diurnal Urgency & $\mathbf{0 . 2 1 9}$ & $\mathbf{0 . 0 3}$ \\
\hline Nocturnal DR \& Nocturnal Urgency & 0.005 & 0.96 \\
\hline
\end{tabular}

$\mathrm{DR}=$ diuresis rate, $\mathrm{ANV}=$ actual number of nightly voids

Table 1. Spearman's rank correlations between diuresis rate, urge perception score and nocturia severity.

\begin{tabular}{|c|c|c|}
\hline Diary Parameters & Spearman's $\rho$ & $\begin{array}{c}\text { Significance } \\
(\mathbf{p})\end{array}$ \\
\hline Nocturnal DR \& ANV & $\mathbf{0 . 4 2}$ & $<\mathbf{0 . 0 0 1}$ \\
\hline Diurnal DR \& ANV & -0.024 & 0.8209 \\
\hline Diurnal DR \& Diurnal Urgency & $\mathbf{0 . 2 1 9}$ & $\mathbf{0 . 0 3}$ \\
\hline Nocturnal DR \& Nocturnal Urgency & 0.005 & 0.96 \\
\hline
\end{tabular}

$\mathrm{DR}=$ diuresis rate, $\mathrm{ANV}=$ actual number of nightly voids 


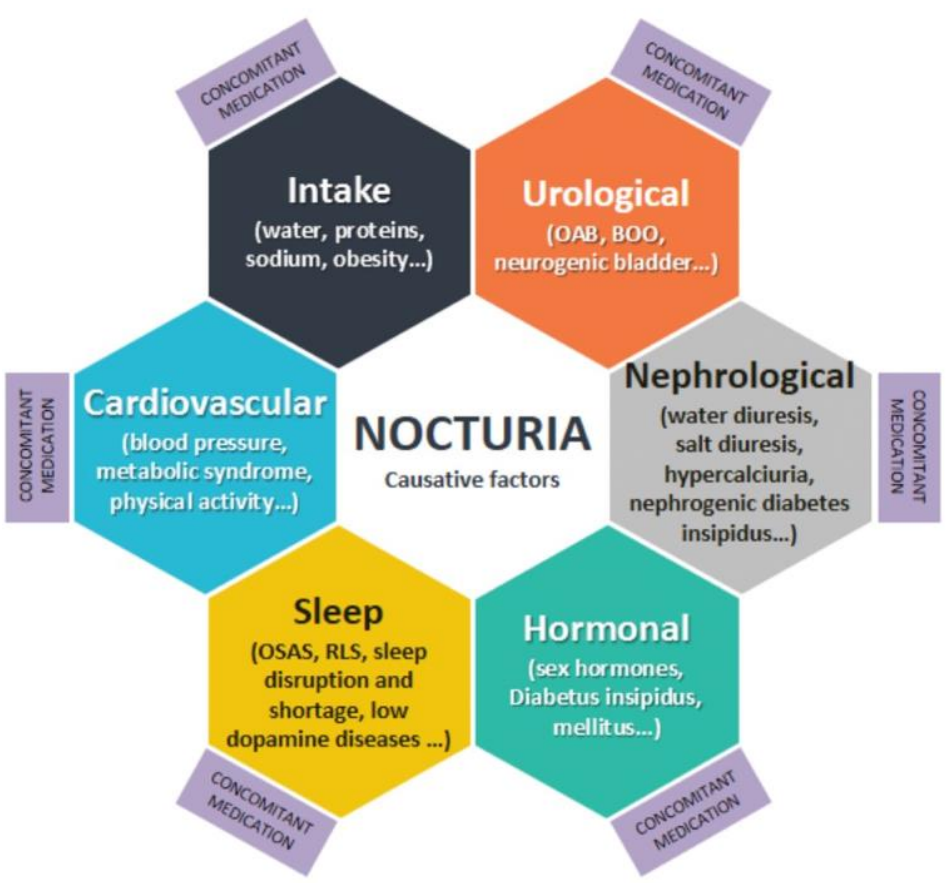

slide_01. 


\section{Distribution of Spearman's Correlation}

Coefficients Between Nocturnal Diuresis

\section{Rates and UPS}

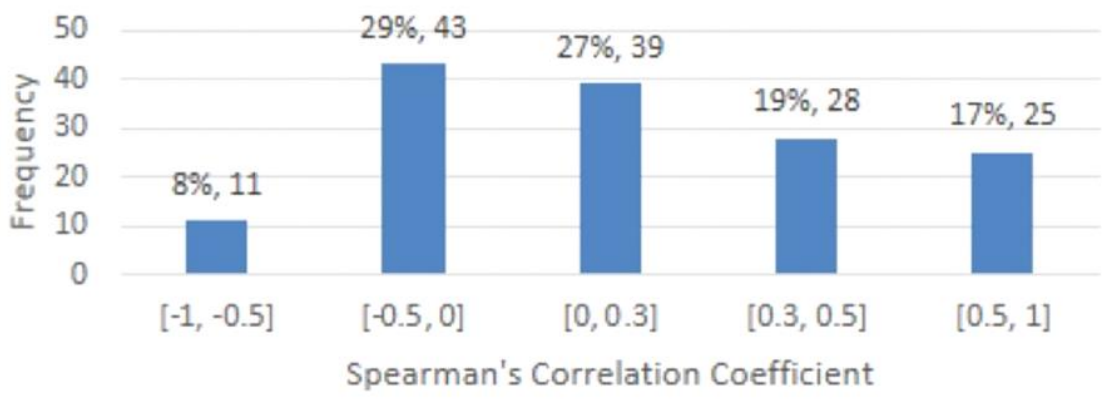

UPS= Urgency Perception Score (with a graded response from 0-4 where 0 is zero desire to void and 4 is a severe urgency)

slide_02. 


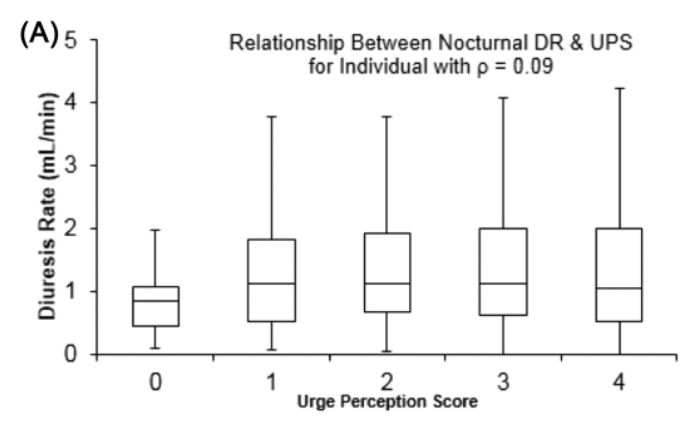

DR: diuresis rate, UPS=Urgency Perception Score

(B) 2,5 Relationship Between Nocturnal DR \& UPS for

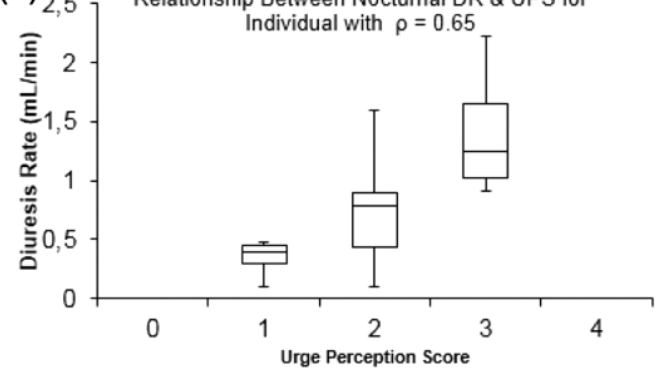

DR: diuresis rate, UPS=Urgency Perception Score

slide_03. 


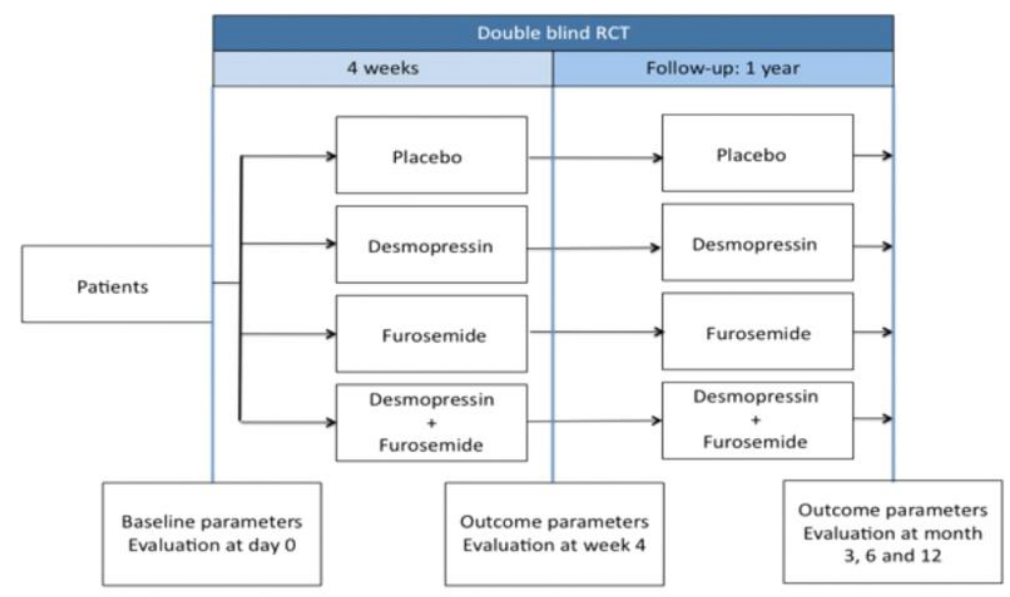

RCT: Randomized Control Trial

slide_04. 


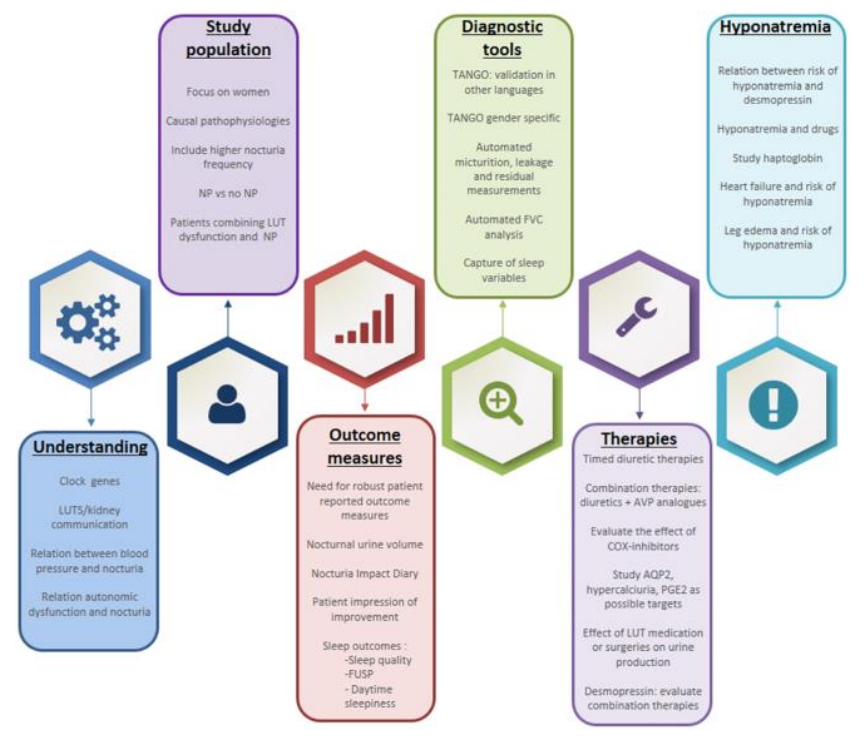

slide_05. 


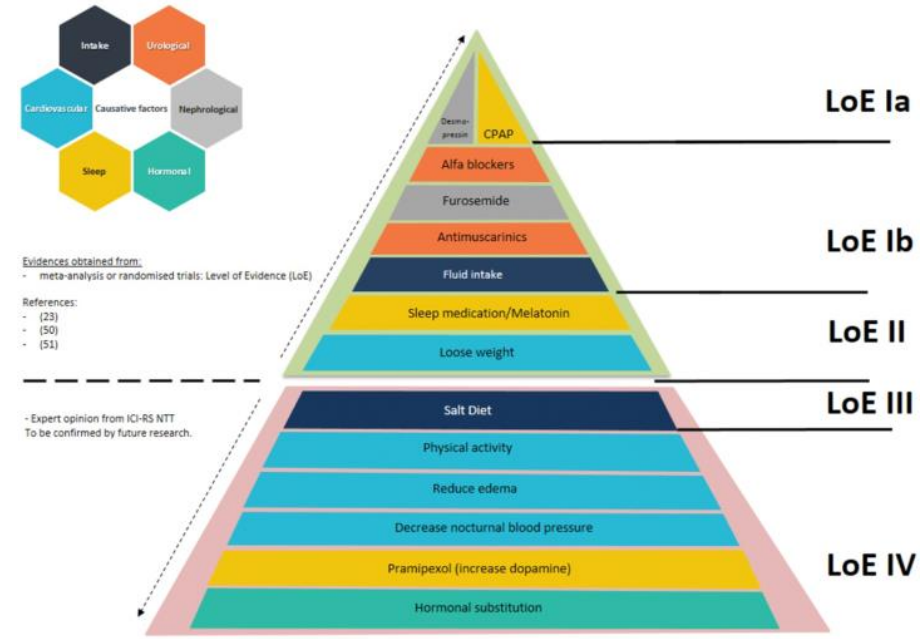

slide_06. 


\section{University Library}

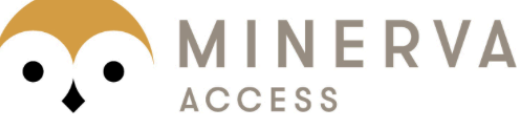

A gateway to Melbourne's research publications

Minerva Access is the Institutional Repository of The University of Melbourne

Author/s:

Everaert, K;Herve, F;Bower, W;Djurhuus, JC;Dmochowski, R;Fine, N;Wein, A;Wyndaele, M;Weiss, J

Title:

How can we develop a more clinically useful and robust algorithm for diagnosing and treating nocturia? ICI-RS 2017

Date:

2018-06-01

Citation:

Everaert, K., Herve, F., Bower, W., Djurhuus, J. C., Dmochowski, R., Fine, N., Wein, A., Wyndaele, M. \& Weiss, J. (2018). How can we develop a more clinically useful and robust algorithm for diagnosing and treating nocturia? ICI-RS 2017. NEUROUROLOGY AND URODYNAMICS, 37 (S4), pp.S46-S59. https://doi.org/10.1002/nau.23569.

Persistent Link:

http://hdl.handle.net/11343/284319 